\title{
Redefining idiomatic writing for the pedal harp
}

Martin Scheuregger

\section{Abstract}

Sounds of shimmering glissandi and resonant arpeggios combine with romantic and angelic imagery to form a stereotype of the modern pedal harp as an instrument capable of a relatively narrow band of expression. Similarly, its fundamentally diatonic design gives rise to issues with chromatic music that its pedal system addresses without fully negating. Taking pitch and timbre as means through which to reconsider the instrument, this paper presents an approach that reflects and celebrates the inherent properties of the harp whilst redefining what idiomatic writing might be. A reappraisal of the instrument's sonic qualities contrasts typical sounds with alternative approaches to timbre. The pitch limitations of the harp inform a new method for localised and structural pitch organisation that is rooted in the pedal mechanism of the instrument. These new modalities and alternative timbres are illustrated with a range of compositional examples throughout.

Keywords:

Harp, composition, set theory, timbre, contemporary music.

Of all western orchestral instruments, the harp ${ }^{1}$ is amongst those most weighed down by unhelpful idiomatic baggage. Its characteristic sounds come easily to mind for composers and listeners alike: shimmering glissandi, resonant arpeggios, etc. Its association with French Romantic - especially Impressionist - music is strong, with composers of the late nineteenth and early twentieth centuries contributing to its solo and chamber music in a way that has come to define the harp's sound. A whole repertoire grew around Debussy's 1915 sonata for flute, viola and harp, the trio becoming the quintessential chamber group for the instrument. The approach to writing for the harp in this repertoire is a product of the aesthetic concerns and stylistic approach of the composers more than it is a deliberate attempt to define the instrument. Debussy and his peers were not creating a manifesto for writing for the harp, but their efforts have undoubtedly had an influence on the works written for the instrument in the succeeding decades. Whilst composers have approached the harp in very different ways since Debussy - especially in the second half of the 
twentieth century and onwards - the associations of the harp with certain sounds has stuck.

The aim here is to provide a conceptual counterweight to commonplace idiomatic harp writing that has its origins in French impressionist music. Common tropes are identified, and conceptually opposing techniques and ideas presented in the context of a new work for solo harp, extracts of which illustrate some of the approaches explored, alongside context from an earlier work for harp and marimba. This practice-based approach brings the compositional process into the centre of the investigation, such that the findings relate to the specifics of the new work whilst also informing more general observations. This process does not provide an all-encompassing alternative to idiomatic writing; instead, my own creative approach takes as its starting point a deliberately antithetical position to the impressionist stereotype. This study explores the compositional possibilities that arise from such a conscious attempt to reframe the character of the harp. This is demonstrated through an aesthetic that is far removed from that traditionally associated with the harp, giving an insight into the capabilities and alternative character of the instrument.

Two complementary approaches separate issues of timbre and pitch, although they ultimately inform one another. The first defines common aesthetic qualities of the instrument so that antithetical descriptions can be given, and timbres realised to achieve them. This process recasts the instrument as ideally suited to fragile textures and hollow sonorities, and embraces resonance. A chromatically nimble instrument is presented, but one which can create a soundworld at odds with the lush resonance of Debussy et al. The second approach focuses on the pitch limitations of the harp, embracing its diatonic roots using fixed pedal positions to generate new modalities that arise directly from the mechanics of the instruments. The intention is to let the materiality of the instrument generate a tonal space that disregards the chromatic dexterity that arose from the instrument's nineteenth-century development, and that was so essential to it finding a place in non-tonal chamber music.

Limitations will be applied in both areas. Issues of timbre will not include any substantial exploration of techniques that require preparation of the instrument, or the use of accessories with which the harp is played. Similarly, the use of pedal positions to create modalities will be limited to the standard tuning of the harp, with the retuning of strings either to accommodate additional pitches or to add microtones not being used in 
the examples presented. Both areas are worthy of further exploration and have the potential - already tapped by some composers - to generate sounds far beyond the standard harp idiom. The possibilities of a more radical approach are addressed in the conclusion of this study, but for the most part the aim is to present an approach to writing for the harp that resits common tropes, and reimagines the instrument in a stylistic frame defined by sparsity, fragmentation, and a concentration on sonority. The intention of this approach, and its self-imposed limitations, is to generate a compositional outlook in which the unidiomatic possibilities of the harp become the very material of the music, the instrument itself defining the content and style of its repertoire.

Notable composers to contribute to similar thinking around the harp include Luciano Berio and Carlos Salzedo. In the case of Berio, his Sequenza II for solo harp brings together myriad techniques with a similar intention to this study although, unlike today, in the year it was completed (1963) the harp's negative Romantic image was relatively unchallenged, and its stereotyped characteristics at odds with the mainstream of contemporary classical - mostly modernist - music (Whatley, 2007, p. 41). In contrast to Berio's approach, Salzedo's earlier work defines a great many techniques that open the instrument up to wider compositional possibilities in a more traditional context (Salzedo, 1921). His aims are similar to Berio's and mine, as he attempts to 'diminish the contempt with which fine musicians regard the harp' (p. 1), but where Berio's work is aggressively anti-Romantic and an extreme counterweight to the harp's stereotype, the results of Salzedo's study are presented in an idiom that still has clear routes in earlier traditions. My intention sits somewhere in the middle, as I aim to find solutions that bring a new sound to the instrument without disregarding its natural qualities, whilst also concentrating more on the qualities of a small array of unified sonorities than either of these predecessors.

\section{Fragmentary sounds: approaches to timbre}

\section{Origins}

The desire to reframe my compositional understanding of the harp began with Be still, for harp and marimba. Composed in its first form in 2012, the work was substantially revised in 2015 with a deliberate attempt to challenge idiomatic writing for each instrument. As a percussionist, I am acutely aware of the clichés that can be relied on when writing for marimba, and in Be still attempted to create music that disregards 
common tropes of its instruments. Although it was important to ignore compositional cliché, I was keen to embrace the natural qualities and materiality of the pair, working with rather than against their inherent qualities and restrictions. The primary method for doing this was to embrace the harp as a naturally sonorous instrument, encouraging the player to damp strings as little as possible, and also to work with the dry tone of the marimba's upper range in spacious high lines, whilst exploiting the subtle resonance lower down.

Be still is made up of ten short movements that are conceptualised as fragments. I have discussed my approach to composing with fragments elsewhere, especially in contrast to miniatures and other forms of brevity (Scheuregger, 2015, 2016), but the important issues in the context of this study are the notion a fragmentary soundworld and aspects of the technical approach to writing for the harp. Be still is characterised by sparse, hollow sounds, and makes no use of the lush, cascading qualities traditionally associated with the instrument. The harp is mostly left to resonate, with some fragments containing limited or no damping. The opening (Figure 1) demonstrates this, the two instruments working together to create successive resonant spaces within which a limited set of pitches is used. The approach to texture is mostly linear, but creates harmonically rich sonorities due to the attention to resonance. In later fragments this is more extreme, with a single pedal position used, avoiding any damping and allowing a seven-note modal pitch space to develop.

\section{INSERT FIGURE 1 HERE}

Figure 1 Be still, I, bars 1-11.

In the fifth fragment, the soundworld is fragile, but subtly changing: the harp is played près de la table, plucked with nails, and muted; the marimba matches these with its own techniques. The constant resonance reinforces the tonal ambiguity rather than providing sonorous affirmation of a key. In the sixth fragment (Figure 2) the fragility of the sound is increased through further timbral devices and imitation of the drier sounding marimba: a muffled major third harmonic is heard, bisbigliando chords mimic the tremolo of the marimba, strings are played with nails and près de la table, and tapping the string in the final gesture creates a hollow sound with an indistinct pitch. The palette of sonorities used across Be still engages with ideas of fragility, delicacy, dislocation, and 
fracturedness, as the work embraces the notion of musical fragmentation in its structure and sound (Scheuregger, 2015, p. 52). Whilst the technical devices used here may be found individually in much harp writing, their combination is not redolent of a traditional approach. It is these ideas that began the process of defining a new harp idiom, and contributed to the earliest part of this study.

\section{INSERT FIGURE 2 HERE}

Figure 2 Be still, VI.

\section{Defining timbres}

The approach to timbre in Be still is broadly to do with three factors: synthesising the complementary sounds of marimba and harp, embracing the harp's resonance, and finding new sonorities in sparse and simple textures. The second and third of these form the starting point for the more explicit exploration of timbre in the new work for solo harp presented in extracts below. Timbre is explored here within the context of several overarching concepts that act as counterweights to some of the common tropes of harp writing. These can be framed as antithetical states, posing a facet of idiomatic writing with its opposite:

- $\quad$ sonorous and resonant $\sim$ dull and muted;

- $\quad$ full and lush $\sim$ fragile and fragmented;

- $\quad$ cascading and flowing $\sim$ tentative and disjointed.

These 'non-idiomatic' states describe a soundworld not usually associated with the harp, and, as they are not technique-specific, are open to myriad interpretations. This step is a useful starting point for composing in a manner that produces results unlike the postDebussy sound described above. Whilst the results of my compositional work do not account for all possible approaches, the departure point might lead others to address these issues, and create different results. As such, the extracts of the new work presented here are intended to be demonstrative rather than prescriptive.

The non-idiomatic descriptions in the above oppositions are interlinked facets of the same basic soundworld, with each suggesting techniques and idioms that can be exploited. Sonorous and resonant sounds are created through carefully spaced chords, 
often with octave reinforcement of important pitches, appropriate use of register, and arpeggiation. 'Full and lush' relates more to texture than to sound, and can be achieved with continuous passagework and rapid patterns of chords - especially repeated revoicings of the same chord - that act to extend the resonance of the instrument, and give the impression of greater volume and depth of sound. Lastly, cascading and flowing movement can be equated to passages of regular broken chords, or the use of the vast array of glissandi uniquely available to the harp, including all major and minor keys, diminished chords, certain dominant sevenths. ${ }^{2}$ All of these techniques are linked, contributing as a whole to an overall sound that has become synonymous with the harp. Setting these descriptions out in the above list makes explicit what is common knowledge to a listener, and common sense to a composer, but by framing them in such a way their opposites can be taken as a starting point for exploring a soundworld at odds with the traditional sound of the harp. The description of dull, muted, fragile, fragmented, tentative and disjointed is not at all redolent of the harp, but instead gives an account of a speculative set of sounds with the potential to create music of a very different nature.

There are facets of basic technique which characterise the standard sound of the harp, and which are used by players to give clarity and fullness capable of balancing with other instruments. By adapting these, a sound that fits this new description can be achieved. Techniques which most influence sound production are primarily to do with playing position, plucking technique, and damping. To create a full sound, the hand is positioned in the centre of the string, the flesh of the fingers and thumb (excluding little fingers) placed on the string, and the strings plucked. By changing any part of this basic technique - playing closer to the soundboard or plucking with nails, for example - the sound will be changed. The possibilities of such changes are explored below in relation to dry and muted sounds, and including new approaches to resonance.

\section{Dry / muted sounds}

The non-typical soundworld described above suggests a range of approaches to dry and muted sounds through different playing techniques. Dry sounds featuring little resonance are the most obvious, being delivered with staccato and etouffée playing techniques in a straightforward manner (see Einarsdóttir, 2013 for the difference in playing technique, and for explanations of other techniques used here). These sounds have clear pitch content, but by changing playing position, dry sounds with different qualities can be 
achieved. Playing close to the soundboard (près de la table) gives a harmonically-rich quality, whilst playing near the top of the strings (haut dans les cordes) creates a thinner sound. These can be exaggerated further by plucking with the fingernails, plectrum or other object, and combined to exaggerate their effect: the sound of a low string plucked with a nail close to the soundboard is metallic and cold, and even when left to vibrate can contribute to the soundworld sought here.

Moving to sounds of less definite pitch opens up further options still. Percussive sounds can be derived from muting strings completely with one hand and playing with the other: a relatively pitchless sound is created by firmly muting the string in the centre, whilst a more resonant sound is achieved by damping the string close to the soundboard in a technique described as xylophonic (Einarsdóttir, 2013). Strings can be played or muted with other objects, and the soundboard itself may be struck, either as a purely percussive effect or in combination with plucking the string to create a Bartók pizzicato. ${ }^{3}$ By applying different techniques in combination, a greater degree of possibilities is opened up, but in all cases resonance is not lost by damping just the sounding strings. Sympathetic resonance is created naturally by both percussive and standard techniques, the force of attack determining the degree of resonance. The pitch relationship between the played and resonating strings is also crucial, with strings vibrating more if those being plucked are tuned to harmonics of the resonating string (particularly octaves, fifths and their compounds). Equally, inharmonic percussive sounds, with little pitch but great volume, will excite the resonance of multiple strings regardless of their tuning. These techniques can be used to form a resonant space in which dry and muted sounds interact. The level to which such resonance is controlled is up to the composer, with approaches ranging from merely instructing the player not to mute strings, to composing with a precise understanding of the sounds being created.

These techniques could be expanded further - Einarsdóttir (2013) offers an extensive guide - but in the context of this study, it is the above effects that have proved most effective, with many of them used in the short extract from the new work presented here (Figure 3). A range of techniques are used as the passage moves from a fully resonant gesture (bars 1-2) to a muted, près de la table sound played with fingernails (bars 1618). Between these, the high register is used melodically (bar 7) with sufficient volume to excite resonance that is heard in the pause of bar 8 . The continuum of sound possible 
between près de la table and normal playing is also exploited (bars 14-15) as are several examples of muting the strings.

In what is a relatively straightforward passage, notational issues already arise. With the wealth of information needing to be communicated, a part has the potential to become confusing, especially in regard to which techniques apply and for how long. The use of brackets is helpful here, whilst established abbreviations and symbols help reduce clutter, but there may still be confusion over muting, which can apply to resonating strings (as in the D-sharp to be muted in bar 4) or to passages which are to be played with one hand and muted with the other (such as bar 9). The notation given by Einarsdóttir for the latter - an open cluster sign over the appropriate range and the instruction 'press firmly' - may be unnecessarily detailed in extended passages in which the muting hand is following the playing hand to effect the change of timbre. Brackets indicating the duration of certain techniques are useful to allay any confusion. Detailed performance directions are needed in these contexts, but a system of symbols may be useful in the future development of this way of writing. Here, I have followed both Einarsdóttir (2013) and Gould (2011).

\section{Resonance}

Un-damped resonance is usually applied to creating clear harmony and in pursuit of a defined sound. Damping is a crucial part of harp technique, and is used to ensure resonance is controlled to suit the music, with different approaches needed for the instrument's different registers. The lower register requires note-by-note damping if a low part is to be heard with clarity, and must be damped in many other situations where the strings resonate sympathetically. The middle register requires damping for the same reasons, although melodic passages can be executed with no damping without creating too muddy a texture, as the resonance in this range gives clarity whilst maintaining a full sound. In the high register, a lack of sustain creates a sound not traditionally used for melodic passages, or else one requiring reinforcement with the middle register.

These are straightforward observations that are both second nature to harpists and self-evident to composers; however, by defining the low register as in need of damping to create clarity, and the high register needing support to give resonance, further counterweights to idiomatic writing can be considered. The low register might be embraced as resonant, muddy and capable of great tonal ambiguity; the high register as 
suited to intentionally hollow sounds as shown in Figure 3 (bar 7). The sympathetic resonance created across the instrument may also be embraced as a desirable feature, rather than avoided in pursuit of clarity. If arising from notes in the same harmonic series, this resonance is broadly consonant; inharmonic, percussive sounds excite more complex, dissonant resonance, which could be expanded with further unmuted sounds in the low register.

\section{INSERT FIGURE 3 HERE}

Figure 3 Extract from new work demonstrating dry and muted sounds.

Allowing the harp to resonate constantly prevents the player from adjusting the pedals without causing pedal buzz, or needing to damp the strings; as such, the chosen pedal setting is very important, as it may be employed for an extended period. Pitch and resonance are therefore intrinsically linked for the harp, and it is by considering the two together that results can be created that approach tonality and timbre in a unified way. This is explored in relation to four-pitch pedal settings below.

\section{New modalities: approaches to pitch}

The harp functions in tonal music thanks to its ability to recreate scales in all possible transpositions through different pedal settings: starting with all pedals in the flat position, one pedal at a time must be moved in the sharp direction to move efficiently through the cycle of fifths. In tonally straightforward music, pedalling may need little consideration by the composer, and although use of chromatic alterations brings complications - a melodic minor scale would, for example, require a change of pedals when changing direction - these are relatively straightforward to negotiate. More advanced tonal music in the nineteenth century, and ultimately expressionist and nontonal music, pose more difficulties; dodecaphonic music for the harp requires extreme care in its composition. For the composer writing music requiring the frequent use of more than seven pitches, planning pedal positions is crucial to success (Adler, 2002, p. 92). The technical facility required of harpists gives composers a fully chromatic instrument for which - with careful planning - the most adventurous music can be written. The calm exterior of the player will hide frantic pedalling beneath the surface, such that we may forget the instrument is essentially diatonic. 
In attempting to re-frame the sonic character of the harp, I have composed with two basic approaches to pitch. The first reflects the above description, treating the instrument as chromatically nimble: such an approach can be seen in examples from Be still, and parts of the new work referenced here, but requires no specific explication. The second approach embraces the harp's essentially diatonic character, and focuses on the creative possibilities offered by considering pitch in terms of fixed pedal positions. The aim here is to move beyond the traditional view of the instrument as having 'pitch problems' and 'pitch assets' (Blatter, 1997, p. 256). Whereas the attitude to timbre detailed above seeks to provide a counterbalance to a sound with its origins in the late nineteenth century, the approach to pitch may be seen as a challenge to compositional methods in which chromatic freedom is paramount.

\section{INSERT FIGURE 4 HERE}

Figure 4 Be still, V.

This approach can be observed in the fifth fragment of Be still (Figure 4), in which the harp uses a fixed pedal position that defines pitch content. A modality is created from this that links the material of the music directly with the materiality of the instrument. The pedal settings create a scale of A-flat harmonic minor with a sharpened fourth, or Aflat Hungarian minor. The clustering of minor seconds (D-E-flat-F-flat) offers rich chromatic potential, and creates a degree of ambiguity as to the tonal centre. In this context, the A-flat does not form an overt melodic centre, although it is a prominent pitch in the fragment. The harp is left to resonate, emphasising the lack of re-pedalling whilst also suggesting modal or drone-based music. This stasis of pitch is at odds with the potential for chromatic dexterity that is so often exploited by composers, as a facet of the harpist's virtuosity, and the composer's inventiveness. Here, the simplicity forms a link with the instrument's ancient predecessors whose designs - and consequent repertoires - would naturally have been very different.

The idea of deliberately limiting the harp to a single pedal setting for a movement is not a radical concept in itself - diatonic music which does not depart from one key would achieve this. However, the approach can create distinctive modalities that are intrinsically linked to the instrument, whilst the harp's unique pedal system creates further idiosyncrasies with creative potential. Moving beyond single pedal positions, 
methods for organising pitch on a structural level can be created that are fundamentally informed by the physicality of the instrument. For example, pitch-class sets separated by a single pedal change may be conceived as related. The use of pedal positions to create fixed modalities, and to inform interrelated modes, are both explored below.

Gotham and Gunn (2016) set out all pitch-class sets available in the 2,187 pedal settings of the harp, giving the number of different settings that create each. This is demonstrated using set theory, so it must be noted that the same prime form may account for different scales: the major and natural minor scales, for example, contain the same intervallic content, so are both classified as $[0,1,3,5,6,8,10]$. This pattern can be generated with 20 different pedal settings, but is not the most prevalent. From Gotham and Gunn's work, many approaches to composing for harp could be taken. The authors conclude by hoping:

that composers will derive from this article a greater understanding of the harp's pitch possibilities that provides at least technical guidance, and perhaps even material inspiration. For instance, some composers may find the effects of individual pedal changes to be a way of guiding successive pitch structures in a piece, or they may find that the relative preponderance of certain sets (and even pitches) make them attractive candidates as core pitch materials for a work. (Gotham and Gunn, 2016, 4.18)

Given my interest in using fixed pedal positions to create modalities, the findings from this article are of particular use, as all possible pitch-class sets available on the harp are given, alongside the frequency of their appearance. Each possible pedal position offers multiple modes, which may in themselves be of great compositional interest. In the interests of brevity, and of identifying some of the more unusual options, pedal settings used here are limited to those containing only four pitches, and the most prevalent set with seven distinct pitches. The ways in which these two groups of pitches are interrogated and applied can be extrapolated to other pitch-class sets to create further compositional possibilities.

\section{Four-note pitch-class sets}


Of the harp's 2,187 possible pedal settings, many are transpositional equivalents, or duplicate the same pitch material exactly. They contain a maximum of seven distinct pitches, and a minimum of four, with scales of fewer than seven pitches - whole tone, pentatonic, etc - containing pairs of adjacent strings duplicating the same pitch enharmonically. This doubling of sounding pitches is one of the characteristic qualities of the harp, allowing for glissandi of certain seventh chords and other scales. On the harp, three pairs of strings can be tuned to the same pitch, leaving the seventh string tuned to a single pitch. Seven possible arrangements of three pairs and a single string can be formed (the single note taking each of the seven strings in turn). When one of these pairs is made up of either the B and C, or E and F strings, one must choose to which pitch the pair or pairs are tuned (B-sharp and C, or B and C-flat, for example). Conversely, pairs of F-G, G-A, A-B, C-D, and D-E can only meet in the 'black notes' that lie between them. In the seven possible arrangements of pairs, four contain either the B-C or E-F pair and one contains both. Where one pair is present, two possible tunings arise; with both pairs, there are four tunings. This gives fourteen possible arrangements of pairs. The final variable is the single string, which may be tuned in a flat, natural or sharp position for each of the fourteen combinations, giving a total of 42 possible pedal positions that give only four pitches in each case.

The characteristics of these 42 tunings are limited to eight possible chords in various transpositions, all of which are seventh chords of one kind or another as outlined in Figure 5. The same prime form is present across pairs of chords that are inversely related: for example, a dominant seventh, which comprises a major third followed by two minor thirds, is the same as a half diminished seventh, which is made of the same intervals in reverse. The labels used by Gotham and Gunn follow Allen Forte's practice of reducing inversely related collections such as these to the same prime form (Forte, 1973), and are maintained for reference, but their different tonal implications is important here.

These pedal settings are most often employed to create tonally useful glissandi: the sound of such an effect on a half diminished or diminished chord will easily come to mind as an idiomatic, even clichéd, effect. Compositional approaches that move beyond such use are considered here.

\section{INSERT FIGURE 5 HERE}

Figure 5 Chords resulting from four-note pedal settings. 
There is much variation in the number of pedal positions which create chords with the same tonic: nine on D-flat down to only one for A. Similarly, certain chord types are more prevalent than others: ten pedal settings will produce minor seventh chords (although each distinct chord can be created by two different settings), whilst only one will create an augmented seventh. The three transpositions of the diminished seventh chord are available in three pedal settings, meaning diminished chords on all twelve pitches are possible. From these observations, a composer may go in various directions. The prevalence of minor seventh chords might suggest an organisation of pitch through shared chord type; alternatively, taking D-flat or E as a tonal centre would give the maximum number of chords sharing the same tonic (each tonic has five distinct chords available, although D-flat has two further pedal settings to reach these). Hierarchies can be created based on the relative prevalence of different factors, and could similarly take into account shared pitches, as seen in the approach to the seven-note modes below.

The compositional possibilities within a fixed pedal setting are also of interest, and may be combined with the interrelationships of different pedal positions to create larger harmonic structures. A range of textural techniques are available due to the pairings of enharmonically equivalent strings: sympathetic resonance becomes more pronounced and may be used creatively, whilst bisbigliando on a single pitch becomes readily available. The pitch material can also be extended through the use of harmonics up to the fifth partial, above which the string must be played too close to or beyond the tuning discs to be feasible (Einarsdóttir, 2013). Those which are reliable are the octave, perfect fifth, double octave, and major third harmonics (the second to fifth partials), extending the pitch material of each pedal setting (although some pitches will be duplicated from the harmonics of different fundamentals). As well as the added pitch material, the use of harmonics gives timbral variety. This is the same with any tuning, but the duplication of pitch classes in four-note pedal settings offers a useful device: two partials of the same fundamental can be heard simultaneously, and multiple instances of the same pitch can be sounded on separate strings. By combining harmonics from different fundamental strings, further effects can be achieved, including microtonal discrepancies of supposed unisons. These effects lend themselves to a spectral organisation of pitch.

\section{INSERT FIGURE 6 HERE}


Figure 6 Pitches available through open strings, fifth harmonics and third harmonics.

The use of octave effects is heard in Berio's Sequenza II, but whereas this is in the context of many other effects and soundworlds, the aim in the extracts of the new work presented here is to employ a greater economy of means and effects. This approach limits the range of possibilities offered to the composer, but presents a clear relationship between pitch material, namely three transpositions of the same four-note set. A unified and resonant soundworld is also created that possess a very distinctive character, as certain pitches appear only as third or fifth harmonics, both of which have a very different tone to each other and to the fundamental open strings. The transposition of material is also, therefore, accompanied by a timbral transformation.

I will restrict the demonstration of four-note pitch-class sets to that which is available in only one pedal position: E augmented seventh, or $[0,2,4,8]$. Here, the four pitches are extended to nine through the use of harmonics as shown in Figure 6. The opening of the new solo harp work uses this material (Figure 7). This is an almost exclusively linear passage, but its effect is mostly harmonic, occasionally melodic. Its combination of harmonics and open strings gives the greater freedom of pitch, but the tendency towards spectral sonorities present in the basic material is embraced, leading towards localised pitch centres of $\mathrm{C}$ and $\mathrm{E}$. The spacing of notes is also led by the harmonic series, something which is exploited thanks to fundamental and spectra being simultaneously available through enharmonically equivalent strings. This is enhanced in passages which focus on specific pitches, and the ability to achieve them with multiple strings (bars 5 and 7). With the technical possibilities leading towards a quasi-spectral texture, timbral devices are used to create further harmonically rich sounds. The combination of près de la table and using the fingernails in the low register emphasises partials other than the fundamental, enhancing the overall sonority. Similarly, sympathetic resonance is used: the effect of the opening is explicit in this regard, using the F-flat string to excite the resonance of the enharmonically equivalent unison E. Here the resonance of the octave harmonic is also exploited next to the F-flat.

This passage sets out an approach to resonance and harmony, and to pitch collections as a function of pedal positions. The observations in relation to timbre and resonance made above are acted upon, whilst leaving room for the piece to develop these further. Similarly, the use of a single pedal position is demonstrated here, whilst the large- 
scale pitch relationships possible through connected four-note pedal settings are left to guide the development as the piece progresses. Given the concentration on timbre and resonance here, the rhythmic notation is kept deliberately vague, using a continuum from empty to black note-heads, through to quavers, semiquavers and grace notes. This gives the player the freedom to vary the tempo in a manner that maintains maximum resonance, but might require experience of playing other music that uses such free notation. ${ }^{4}$

\section{INSERT FIGURE 7 HERE}

Figure 7 Opening of new solo harp work using the E augmented seventh $[0,2,4,8]$ pedal setting. 5

\section{Commonly occurring pitch-class sets}

The most prevalent pitch-class set arising from multiple pedal settings 'does not correspond to any scale in common usage' (Gotham and Gunn, 2016, 7): it occurs 90 times but contains only six pitches. It does in fact create Scriabin's 'Mystic chord' $[0,1,3,5,7,9]$, a collection that can be used melodically to great effect. However, the most common set to occur with seven distinct pitches is achievable with 40 pedal settings. With a prime form of $[0,1,2,4,7,8,9]$, these settings produce two possible scales, with an interval sequences in semitones of 1-1-3-2-1-1-3 and 1-1-2-3-1-1-3. These are inversely related - the intervals of one can be reversed to create the other - so are classified as the same pitch-class set (see Forte, 1973). The two resultant modes are shown in Figure 8, beginning on middle $\mathrm{C}$ : they can be rotated to begin at a different point of the interval sequence, but these will provide the basic form here. To move from one to the next, the inversional relationship can be disregarded: the change can be seen simply as raising the middle pitch of mode one by a semitone to reach mode two. If used in a serial setting, mode one is P0, or the un-transposed prime, mode two is RI9, or a retrograde inversion transposed up nine semitones.

\section{INSERT FIGURE 8 HERE}

Figure 8 Mode 1 and mode 2 of set 7-20. 


\section{INSERT FIGURE 9 HERE}

Figure 9 Mode 1 on C-sharp.

Further observations can be made about these modes in relation to the harp and its melodic and harmonic possibilities, for which reference to the scale based on C-sharp (as that based on $C$ is not available as a pedal setting) will be made (Figure 9). This mode is made interesting by its symmetry around F/F-sharp. Two three-note chromatic clusters $([0,1,2]$ trichords $)$ sit either side of this point, one centred around $D$, the second around A. Both pitches of the centre point ( $\mathrm{F}$ and F-sharp) are not present in one mode; instead, one is found in the first, the other in the second. In tonal terms, this generates either a minor or major triad on the second degree of the scale, in this case D, with chromatic clusters around the tonic and fifth, as shown in Figure 10. This is merely a way of rationalising a pitch collection, and other descriptions may be equally valid, but in arranging it in this way, compositional possibilities are suggested.

\section{INSERT FIGURE 10 HERE}

Figure 10 Pitch organisation within mode 1 and mode 2.

Maintaining the same pedal position but changing the starting note gives six more modes, but unlike the changing feel that can be achieved by playing a major scale starting on a different scale degree - giving the seven Church modes - there is a less definable change here, the two chromatic trichords resisting any strong aural relationship to a tonic. Nevertheless, a tonal centre may be found in any of the seven pitches. The two trichords are related through pairs of perfect fifths a semitone apart, offering various possibilities that may be exploited by a composer. Indeed, fifths are used to outline a pitch space with wide possibilities in the extract from the new work below (Figure 14).

The two modes are available in ten transpositions each, those starting on $\mathrm{C}$ and $\mathrm{G}$ being unavailable due to the enharmonic limitations of the instrument, and the presence of semitone clustering. Amongst the available transpositions, several contain adjacent $\mathrm{C}$ and $\mathrm{B}$, and/or $\mathrm{E}$ and $\mathrm{F}$ strings in the natural position: in these cases, altering certain pedal settings will produce no change in the pitch material, but will create a re-entrant tuning (one in which an ascending or descending pattern is interrupted by a change in direction). 
These enharmonic equivalents account for the doubling from two lots of ten transpositions, to 40 total pedal arrangements. Figure 11 shows all possible tunings, with enharmonically equivalent pedal settings given in smaller staves.

The richly interrelated nature of these modes offers a wide range of compositional options, whilst their derivation from pedal positions ties them to the physicality of the harp itself. Although they can be defined in analytical terms, their origin in pedal positions creates a link that is both definite and abstract. In taking these modes as the compositional basis for part of the new work presented here, there are two challenges which I hope to address. The first is to show the compositional possibilities of working in a mode derived from the physicality of the instrument; the second, to use the modes in different transpositions to create music which begins to exploit interrelationships of the modes. The latter relies on relationships between transpositions being defined, something which can be achieved by considering the modes in a cycle.

\section{INSERT FIGURE 11 HERE}

Figure 11 Mode 1 and mode 2 of set 7-20 in all available transpositions.

\section{INSERT FIGURE 12 HERE}

Figure 12 Mode 1 in transpositions possible on the harp, tonic pitches with hollow noteheads.

A cycle of each mode can be generated which places transpositions that share the most pitches next to each other. In Figure 12, these are shown for mode one, with similar pitches aligned vertically. Unlike the major scale, for which a transposition can be accomplished with the change of only one pitch, in both modes here a minimum of two pitches must be changed to affect a transposition. Similar to the major scale, the closest transpositions are a fifth away, generating a circle of fifths in which neighbouring modes share five of their seven pitch classes. However, the cycle does not continue a straightforward pattern, in which distance from the home mode relates to a greater difference in constituent pitches. For sake of completeness, all twelve transpositions of the modes can be considered, although those beginning on $\mathrm{C}$ and $\mathrm{G}$ are not available as pedal settings on the harp. From any one mode, by moving through the cycle of fifths, the number of pitches that differ from the home mode form the pattern $2,4,4,3,3,3$. The 
final 3 represents the opposite point on the circle, from which the pattern is reversed. As a result, the potential compositional connections between modes can be reordered, such that modes a fifth away from the tonic are the closest; followed by a major third, a minor second, and a tritone; and finally by a major second, and a minor third (see Figure 13). Like that of the tonal circle of fifths, the underlying process remains transpositional, but the presence of two $[0,1,2]$ trichords creates much repetition of pitches, and accounts for the non-linearity of some relationships.

\section{INSERT FIGURE 13 HERE}

Figure 13 Relationships between modes in terms of changes in pitch content.

The result of these relationships is dependent on the approach of the composer. Considering each pedal setting as a mode (with a defined tonic) opens the possibility of creating connections between tonal centres as outlined here. By conceiving them as pitch collections in a non-tonal space, changes between pedal settings may be a way of moving between linked collections without the baggage of a tonal centre: in this scenario, the interplay of modes one and two, and the recurrence of chromatic clusters, may be of compositional interest. In both cases, the pitch material has been derived from the physicality of the instrument itself, and although the music that this creates may sound no different to any other for the harp, its technical foundation in the instrument's pedal mechanism gives a strong aesthetic link between instrument and musical content.

\section{INSERT FIGURE 14 HERE}

Figure 14 Extract of new work demonstrating seven-note modes.

In the final extract of the new work presented here (Figure 14), modes one and two on C-sharp then G-sharp are used. The opening gesture presents the mode in a form in which the scalic relationship between pitches is not emphasised. Instead, fifths predominate to generate a large, tonally ambiguous pitch space. The eight notes of bars 1-2 have the gestural quality of a cycle of fifths, even when the intervals change, suggesting an endless cycle that is logically impossible with only seven pitches. This tension aims to give the impression of a greater freedom of pitch material than is actually 
present. This is helped by considering modes one and two as being related, so that the $\mathrm{F}$ or F-sharp can be used freely to create further fifths. A modulation to the modes on Gsharp is achieved by treating the shared pitches of the two transpositions as a pivot point (bar 14). Although only two pitches change, the E-flat becomes a D-sharp, requiring three pedal changes in total. This pivot chord has the practical advantage of allowing the player to change pedals whilst maintaining resonance, and demonstrates how the physicality of the instrument can further determine the use of pitch. Indeed, the concept of a pivot chord is borrowed directly from tonal music, used here for the same purpose, but is arrived at quite differently. The modulatory passage (bars 11-16) also takes advantage of the semitone clusters, the shared trichord forming a transition section framed by the other trichords as shown in Figure 15.

\section{INSERT FIGURE 15 HERE}

Figure 15 Modal modulation using pivot chords and clusters.

\section{Conclusions}

This study has presented a selection of approaches to the re-thinking of idiomatic harp writing. These have been focused around timbre and pitch, with abstract ideas given alongside applied examples in three extracts from a new work for solo harp. The aim has been to counterbalance stereotyped sounds with new timbral ideas, re-examining the natural qualities of the instrument. The materiality of the harp is embraced, with approaches to pitch that derive from the idiosyncratic pedal mechanism, and treating its limitations as creative tools. Brought together, these two areas reflect an approach that is concerned with engaging with what is natural to the harp, whilst avoiding cliché, and ultimately re-defining what idiomatic writing might be.

The approach to timbre has given a rich soundworld that is unified by a shared set of descriptions that oppose the traditional associations of the harp: dull, muted, fragile, fragmented, tentative, and disjointed. Ranging from unusual percussive sounds to subtly inflected standard techniques, the variety of sonorities is demonstrated in the short extracts from the new work, whilst leaving many options open. Resonance has been approached by considering the implications of basic harp technique, and how this relates to the different registers, with the possibilities of muddied low resonance suggested, and

the sounds of harmonics and sympathetic resonance demonstrated. By relating 
resonance to pedal settings, a range of interesting effects that take advantage of four-note tunings have been shown, with the emphasis on the sonic character that can be achieved when only one such pedal setting is used. The possibility for four-note pitch-sets to be used to create more complex, interrelated structures, has been demonstrated, and reflects a generalised principle that could be applied to other such settings. At the other end, the most prevalent, seven-note pitch-class set has been considered in terms of interrelated modes, which further develop the idea of large-scale pitch relationships that are tied to the physicality of the harp.

Finally, this study raises more general issues around idiomatic writing for instruments. The approach to challenging idiomatic writing here has created intentionally subtle results. Typical timbres have been defined and countered in ways which do not radically extend a player's fundamental technique, or introduce new objects to the instrument. The result is a sound that does not conform to traditional idioms, but neither does it radically alter the harp's fundamental sound. The re-examination of pitch produces sounds which are also broadly familiar: no retuning of individual strings is used, either to extend the seven-note limit, or to introduce microtones. Instead, the underlying connection with the harp's pedal mechanism provides a conceptual counterweight to traditional practice. Overall, the approach taken can be characterised as an attempt to embrace and redefine the natural qualities of the harp. A different approach to idiomatic writing could have been used, treating the instrument as a completely blank canvas, and finding techniques which produce sounds without any reference to established techniques. Both this approach and my own represent valid strategies for redefining idiomatic writing - as would the many approaches in between but each has its own aesthetic context that is fundamentally linked with the composer. There can be no approach which is completely neutral in this regard: the practice-led method of this study is the only useful one that can be taken, and the idiosyncratic results of different practitioners must be embraced. Echoing Gotham and Gunn's desire for their work to be useful for composers $(2016,4.18)$, so my aim here has been to demonstrate one approach and to open the door for others.

\section{Notes on Contributor}


Dr Martin Scheuregger is a Senior Lecturer in Music at the University of Lincoln, where he leads the BA (Hons) Music programme. He completed a PhD combining musical analysis and composition at the University of York in 2015, funded by the Arts and Humanities Research Council. He is currently investigating the methodologies and cultures of composition-based practice-research, focusing on the interplay between autoethnographic, analytical and other approaches, and more broadly examining the impact of neoliberal research culture on creative practice in the academy. Other projects include an extensive study of the music of British composer George Benjamin, and ongoing work on temporality and the perception of time in contemporary classical music.

\section{Bibliography}

Adler, S. 2002. The study of orchestration (3rd ed.). New York: WW Norton.

Berio, L. 1963. Sequenza II (score). Budapest: Universal Edition.

Blatter, A. 1997. Instrumentation and Orchestration. Belmont: Schirmer, Thomson Learning.

Einarsdóttir, G. 2013. The harp in contemporary music. (PhD dissertation). University of the Arts Helsinki, Sibelius Academy. http://sites.siba.fi/web/harpnotation Forte, A. 1973. The structure of atonal music. New Haven: Yale University Press.

Gotham, M. R., \& Gunn, I. A. 2016. Pitch Properties of the Pedal Harp, with an Interactive Guide. Music Theory Online, 22(4).

http://mtosmt.org/issues/mto.16.22.4/mto.16.22.4.gotham.html

Gould, E. 2011. Behind Bars: The Definitive Guide to Music Notation. London: Faber Music.

Salzedo, C. 1921. Modern study of the harp. New York: G. Schirmer.

Scheuregger, M. 2015. Conceptions of Time and Form in Twentieth and Twenty-firstcentury Music. (PhD dissertation). University of York.

Scheuregger, M. 2016. Responding to the modernist fragment. Textual Practice, 30:1, 310. doi:10.1080/0950236X.2015.1119982 
Whatley, K. 2007. Rough Romance: Sequenza II for Harp as Study and Statement. In J. K. Halfyard (Ed.), Berio's Sequenzas (pp. 39-52). Aldershot: Ashgate.

\footnotetext{
1 'Harp' is used throughout to refer to the pedal harp standard in Western concert music. 2 See Blatter, 1997, p. 256 for a list of dominant sevenths that can be performed without additional pitches as glissandi (through enharmonic doubling of pitches) and those which can be achieved as dominant ninths.

${ }^{3}$ On the harp, a Bartók pizzicato is similar in sound to that of bowed string instruments, but necessarily has a very different technique: "The finger plucks the string close to the sound board and then immediately slides off the string to hit the sound board.' (Einarsdóttir, 2013)

${ }^{4}$ Many works by Kurtág and some by Feldman, for example.

5 The use of diamond noteheads to indicate harmonics other than the octave are taken from Einarsdóttir (2013).
} 